\title{
Contribuição dos cocolitoforídeos para o aporte de carbonato de cálcio durante o Último Máximo Glacial na Margem Continental Sul Brasileira Contribution of the coccolithophores to the input of calcium carbonate during the Last Glacial Maximum on the Southern Brazilian Continental Margin
}

\author{
Juliana de Freitas Gonçalves ${ }^{\text {ab }}$, Adriana Leonhardt ${ }^{\text {ac }}$ \\ ${ }^{\text {a } U n i v e r s i d a d e ~ F e d e r a l ~ d o ~ R i o ~ G r a n d e ~}$ \\ boncalvesjuliana.f@ gmail.com, ${ }^{\mathbf{c}}$ adriana.leonhardt@yahoo.com.br
}

\begin{abstract}
Resumo
O ciclo do carbono durante o período glacial apresenta uma oportunidade única para a investigação de uma condição climática absolutamente distinta da atual. O conhecimento acerca da última glaciação pode ajudar a entender o clima atualmente e as potenciais mudanças ambientais futuras. A sensibilidade dos cocolitoforídeos a mudanças na química da água superficial possui grande relevância para os ciclos biogeoquímicos e como resposta para mudanças no sistema climático. O presente estudo utilizou um testemunho de piston core localizado nas coordenadas $47^{\circ} 18^{\prime} 28^{\prime \prime} \mathrm{W}, 29^{\circ} 24^{\prime} 19^{\prime \prime} \mathrm{S}$, retirado do talude da Bacia de Pelotas a fim de analisar a importância dos cocolitoforídeos no aporte de carbonato de cálcio $\left(\mathrm{CaCO}_{3}\right)$ para o fundo marinho na região do Cabo de Santa Marta. O testemunho compreende o intervalo entre 18,16 mil anos (ka) e 40,61 ka, incluindo o Último Máximo Glacial (UMG) e o início da deglaciação. Foram quantificados no mínimo 300 cocólitos e 5 campos de visão ao microscópio por amostra para identificação das espécies de cocolitoforídeos. Foram realizadas quatro datações através da técnica do ${ }^{14} \mathrm{C}$ ao longo do testemunho. O conteúdo de $\mathrm{CaCO}_{3}$ nos sedimentos finos foi obtido através de ataque por ácido clorídrico. Os maiores valores de carbonato nos sedimentos ocorrem durante a deglaciação, porém a maior contribuição dos cocolitoforídeos para o aporte de $\mathrm{CaCO}_{3}$ parece ocorrer durante o UMG, quando comparadas as curvas de conteúdo de carbonato e a curva de abundância absoluta das espécies. As espécies que mais contribuíram para o aporte de carbonato foram Emiliania huxleyi, Gephyrocapsa spp. e Florisphaera profunda.
\end{abstract}

Palavras-chave: Cocolitoforídeos; Último Máximo Glacial; Carbonato marinho.

\begin{abstract}
The carbon cycle during the glacial period presents an unique opportunity to investigate climatic condition distinct from the present. The knowledge about the last glaciation might help to understand current climate and potential future environmental change. The coccolithophores sensitivity to modification in the chemestry of shallow waters has great relevance to biogeochemical cycles and as feedback to change in the climatic system. This study used a core located at $47^{\circ} 18^{\prime} 28^{\prime \prime} \mathrm{W}, 29^{\circ} 24^{\prime} 19^{\prime \prime}$ $\mathrm{S}$, in the slope of the Pelotas Basin. The cores was used to analyze the importance of coccolithophores on the input of calcium carbonate $\left(\mathrm{CaCO}_{3}\right)$ to the seabed in the Santa Marta Cape region. This core comprises the interval between 18,16 thousand years (ka) and 40,61 ka, including the Last Glacial Maximum (LGM) and the outset of the deglaciation. At least 300 coccoliths and 5 vision fields were quantified under the microscope in each sample to identify the coccolithophores species. Four samples were dated using the ${ }^{14} \mathrm{C}$ technique over the core. The $\mathrm{CaCO}_{3}$ content in fine sediments were obtained through acid attack using hydrochloric acid. The carbonate highest values in sediments occur during the deglaciation, however the larger contribution of the coccolithophores to the input of $\mathrm{CaCO}_{3}$ seems to occur during the LGM compared to the carbonate content curve and the curve of absolute abundance of species. The species contributing most to the carbonate input were Emilianiahuxleyi, Gephyrocapsa spp. e Florisphaera profunda.
\end{abstract}

Keywords: Coccolithophores; Last Glacial Maximum; Marine carbonate.

\section{Introdução}

O estudo do ciclo do carbono durante o período glacial apresenta uma oportunidade única para investigar uma condição climática absolutamente distinta da atual. O conhecimento acerca da última glaciação pode ajudar a entender o clima atualmente e as potenciais mudanças ambientais futuras Heinze (1994). O Último Máximo Glacial (UMG) pode ser definido como o intervalo em que a cobertura de gelo global atingiu o seu volume máximo durante a última 
glaciação. A cronozona do UMG foi definida por Mix et al. (2001), como o intervalo entre 19 e 23 mil anos (ka) antes do presente (AP). As modificações climáticas do UMG refletiram-se fortemente no sistema atmosfera-oceano.

Por ser considerado o maior grupo de organismos produtores de carbonato de cálcio pelágico no planeta, os cocolitoforídeos possuem um papel importante no ciclo do carbono marinho. A sensibilidade dos cocolitoforídeos a mudanças na química da água superficial possui grande relevância para os ciclos biogeoquímicos e como resposta para mudanças no sistema climático, O’Dea et al. (2014). A calcificação dos cocólitos pode ser influenciada por mudanças no ciclo global do carbono, as taxas de calcificação de cocolitoforídeos podem diminuir sob uma condição de aumento nas concentrações de $\mathrm{CO}_{2}$ atmosférico, Baumann et al. (2004).

Juntamente com os foraminíferos planctônicos, os cocolitoforídeos são os principais responsáveis por criar e manter o gradiente vertical da alcalinidade na água do mar, Rost \& Riebesell (2004). No Atlântico Sul, o carbonato derivado dos cocolitoforídeos domina os giros oligotróficos, enquanto que o carbonato proveniente dos foraminíferos planctônicos aumenta consideravelmente em áreas mais férteis e mesotróficas, como a zona de divergência equatorial, Baumann et al. (2004). Assim, potenciais respostas para o aumento do $\mathrm{CO}_{2}$ atmosférico, que poderia resultar de mudanças na fotossíntese e também na calcificação, como também na mudança na dominância dos cocolitoforídeos, podem ser cruciais na tentativa de prever mudanças climáticas futuras, Rost \& Riebesell (2004). O presente estudo visa avaliar a importância da contribuição dos cocolitoforídeos para o aporte de carbonato de cálcio para o fundo oceânico durante o Último Máximo Glacial, na região do Cabo de Santa Marta.

\section{2. Área de Estudo}

O testemunho estudado foi recuperado do talude da Bacia de Pelotas, localizado nas coordenadas $47^{\circ} 18^{\prime}$ $28^{\prime \prime} \mathrm{W}, 29^{\circ} 24^{\prime} 19^{\prime \prime} \mathrm{S}$ nas proximidades do Cabo de Santa Marta (figura 1). A Bacia de Pelotas corresponde ao segmento meridional das bacias marginais que compõem a Margem Continental Brasileira. Estende-se por $210.000 \mathrm{~km}^{2}$ até a isóbata de $2000 \mathrm{~m}$ entre os paralelos $28^{\circ} \mathrm{S}$ e $34^{\circ} \mathrm{S}$. Apoiada sobre um embasamento composto pelo complexo cristalino do pré-Cambriano e pelas seqüências sedimentares e vulcânicas, paleozóicas e mesozóicas da Bacia do Paraná, Dias et al. (1994).

O clima da área de estudo está sob o controle do centro de alta pressão do anticiclone do Atlântico Sul. A Alta Subtropical do Atlântico Sul (ASAS) é um sistema de alta pressão localizados em torno de $30^{\circ} \mathrm{S}$ de latitude sobre o oceano Atlântico. Está associada à circulação média meridional da atmosfera pela célula de Hadley, Wainer \& Taschetto (2006). Variações de intensidade e posição da Alta Subtropical afetam diretamente o clima na América do Sul, e particularmente, do Brasil. A ASAS é responsável pela predominância dos ventos de NE na região sudoeste ao longo do ano, Bastos \& Ferreira (2000), seguido dos ventos SW durante a passagem das frentes frias que são mais comuns no inverno do que no verão.

Referindo-se à circulação oceânica, a área de estudo sofre a influência da Corrente do Brasil (CB). A CB é a corrente de contorno oeste associada ao Giro Subtropical do Atlântico Sul. Tem origem aproximadamente em $10^{\circ} \mathrm{S}$, compreendendo o ramo sul da Corrente Sul Equatorial (CSE) que se bifurca ao chegar a costa brasileira, formando também a Corrente Norte do Brasil (CNB). A CB flui para o sul contornando a América do Sul até chegar na região da Confluência Brasil-Malvinas. Transporta a Água Tropical (AT) na superfície e a Água Central do Atlântico Sul (ACAS) na região da picnoclina. A CB torna-se mais profunda e com maior transporte em direção ao sul.

A área de estudo é também fortemente influenciada pela pluma do Rio da Prata. Esta pluma apresenta um deslocamento sazonal, atingindo a região do Cabo de Santa Marta a aproximadamente $28^{\circ} \mathrm{S}$ durante o inverno austral, e sofre uma retração alcançando os $32^{\circ} \mathrm{S}$ no verão do hemisfério sul, Piola et al. (2005). A baixa salinidade das águas do Prata afeta a circulação, a estratificação e a distribuição de nutrientes e espécies biológicas sobre esta vasta região na plataforma continental adjacente, Möller et al. (2008). O input do Rio da Prata no oceano é um importante vetor de exportação de carbono proveniente do continente, Degens et al. (1991).

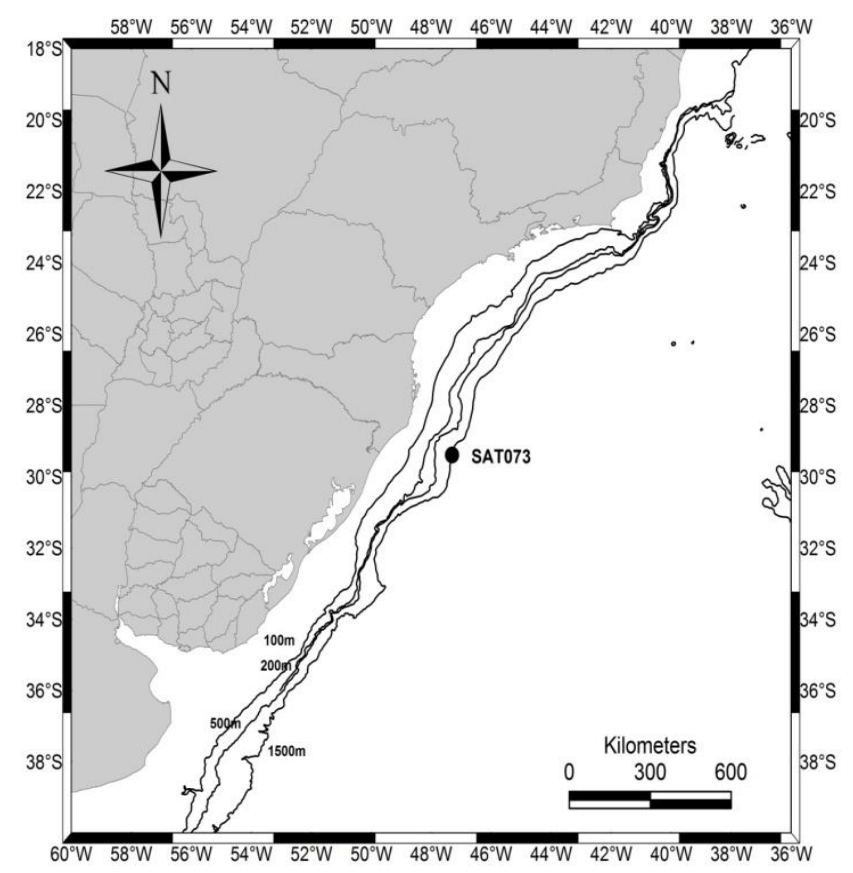

Figura 1. Localização do testemunho SAT-073. 


\section{Material e Métodos}

O testemunho SAT-073 foi doado pela Agência Nacional do Petróleo (ANP). A coleta do testemunho foi realizada através de um testemunhador do tipo piston core, no talude da Bacia de Pelotas, a uma profundidade de lâmina d'água de $1613 \mathrm{~m}$, com recuperação de $2,25 \mathrm{~m}$ de sedimentos. Salienta-se que $20 \mathrm{~cm}$ de sedimentos no topo do testemunho foram retirados pela empresa, antes de sua doação.

A amostragem nos testemunhos foi realizada a cada $3 \mathrm{~cm}$, com auxílio de seringas de $2 \mathrm{~cm}$ de diâmetro com as pontas removidas, permitindo a estimativa do volume da amostra. As amostras foram pesadas ainda frescas e depois secas em estufa $\left(50^{\circ} \mathrm{C}\right)$. Os sedimentos foram peneirados em malhas de $63 \mu \mathrm{m}$ e de $125 \mu \mathrm{m}$. Para este trabalho, somente a porção superior do testemunho foi analisada, totalizando 18 amostras.

\subsection{Análises Radiométricas}

Foram realizadas quatro datações de radiocarbono nas profundidades de $25 \mathrm{~cm}, 40 \mathrm{~cm}, 61 \mathrm{~cm}$ e $70 \mathrm{~cm}$, conduzidas em amostras de foraminíferos planctônicos da espécie Globigerinoides ruber (fração $>150 \mu \mathrm{m})$. O peso mínimo utilizado para este tipo de análise é de 5 miligramas. As análises foram realizadas no Keck Carbon Cycle Accelerator Mass Spectrometry Facility, da Universidade da California. As profundidades foram determinadas de acordo com a curva do $\delta^{18} \mathrm{O}$, a fim de demarcar os diferentes intervalos apontados pela razão isotópica de oxigênio. As demais idades foram calculadas por interpolação linear devido à alta resolução temporal constatada no testemunho.

Os resultados das datações absolutas foram corrigidos para o efeito reservatório e calibradas para anos calendário através do Calib REV7.1.0 Program, Stuiver \& Reimer (1993). Para a região de estudo, o efeito reservatório é de 439 anos e o $\Delta \mathrm{R}=39 \pm 60$.

\subsection{Conteúdo de $\mathrm{CaCO}_{3}$}

A análise dos teores de carbonatos nos sedimentos finos foi realizada no Núcleo de Oceanografia Geológica da Universidade Federal do Rio Grande (FURG). Foram utilizadas 1-2 g de sedimento seco da fração $<63 \mu \mathrm{m}$ para cada amostra, pesadas em balança analítica de 0,0001 g de precisão (inicial). O conteúdo de carbonato nessas amostras foi quantificado através de digestão ácida destes sedimentos com $\mathrm{HCl}(15 \mathrm{~mL}$, diluído a 10\%), realizada em capela. Este ácido reage com os carbonatos de acordo com a equação abaixo (descrita para o carbonato de cálcio, o carbonato mais comum nos sedimentos marinhos):

$$
4 \mathrm{HCl}+2 \mathrm{CaCO}_{3}=2 \mathrm{CaCl}_{2}+2 \mathrm{H}_{2} \mathrm{O}+2 \mathrm{CO}_{2}
$$

Durante o andamento da reação, uma efervescência típica é observada, oriunda da liberação do $\mathrm{CO}_{2}$. Quando esta não foi mais observada, considerou-se que a reação havia chegado ao fim.

Após a digestão ácida, as amostras foram lavadas várias vezes para eliminar possíveis resíduos de $\mathrm{HCl}$ e $\mathrm{CaCl}_{2}$, e secas em estufa à $60^{\circ} \mathrm{C}$. As amostras foram novamente pesadas (peso final) a fim de avaliar o conteúdo de carbonato, conforme se segue:

$$
\text { Carbonatos }=\frac{\text { peso inicial }+ \text { peso final }}{\text { peso inicial }} \times 100
$$

\subsection{Análise dos cocolitoforídeos}

Foram quantificados no mínimo 300 cocólitos e 5 campos de visão por amostra. As lâminas foram observadas em microscópio petrográfico com aumento de 1000 x com objetiva em óleo de imersão. As espécies de cocolitoforídeos foram identificadas segundo Antunes (2007).

\subsection{Abundância Absoluta}

A abundância absolta de cocólitos foi calculada, segundo a fórmula proposta por Koch \& Young (2007):

Onde:

$$
\frac{\mathrm{A} * \mathrm{~N}}{\mathrm{f} * \mathrm{n} * \mathrm{~W}}
$$

A= Área da lamínula;

$\mathrm{N}=$ Número de total de cocólitos contados na amostra; $\mathrm{f}=$ Área de um campo de visão; $\mathrm{n}=$ Número de campos de visão contados; $\mathrm{W}=$ Peso do sedimento na lamínula.

\section{Resultados e Discussão}

\subsection{Cronologia}

A tabela 1 apresenta as idades de ${ }^{14} \mathrm{C}$ obtidas nas amostras selecionadas e as idades calibradas após a correção para o efeito reservatório. As idades para as demais profundidades foram calculadas por interpolação linear. A amostra do topo apresenta uma idade bastante alta, evidenciando a retirada dos $20 \mathrm{~cm}$ no topo do testemunho realizada pela empresa.

Tabela 1: Profundidade das amostras enviadas para datações de

\begin{tabular}{|c|c|c|c|}
\hline $\begin{array}{l}\text { Profundidade } \\
\text { (cm) }\end{array}$ & $\begin{array}{l}\text { Idade } \\
{ }^{14} \mathrm{C} \\
\text { (anos } \\
\text { AP) } \\
\end{array}$ & $\begin{array}{l}\text { Idade }{ }^{14} \mathrm{C} \text { corrigidas } \\
\text { para o efeito } \\
\text { reservatório (anos } \\
\text { AP) }\end{array}$ & $\begin{array}{l}\text { Idade }{ }^{14} \mathrm{C} \\
\text { calibrada } \\
\text { (anos } \\
\text { AP) }\end{array}$ \\
\hline 25 & 15460 & 15021 & 18245 \\
\hline 40 & 15850 & 15411 & 18676 \\
\hline 61 & 16770 & 16331 & 19721 \\
\hline 64 & 26510 & 26071 & 30332 \\
\hline 67 & 29200 & 28761 & 32849 \\
\hline 70 & 31920 & 31481 & 35388 \\
\hline
\end{tabular}
radiocarbono e respectivas idades calibradas para o testemunho SAT073.

\subsection{Conteúdo de $\mathrm{CaCO}_{3}$}

O conteúdo de carbonato encontrado na fração fina dos sedimentos do testemunho SAT-073 variou entre $23,05 \%$ a $42,42 \%$. Entre 37,91 ka e $18,98 \mathrm{ka}$, os valores de carbonato são mais baixos, aumentando a partir desse ponto, após o UMG. Os maiores valores para o carbonato aparecem no topo do testemunho entre 18,5 ka e 18,16 ka. Os períodos de maior conteúdo de carbonato estariam relacionados a intervalos mais quentes Crowley (1983), o que, neste testemunho, correponde à deglaciação. 
A relação entre a abundância absoluta total de cocólitos e o conteúdo de $\mathrm{CaCO}_{3}$ (figura 2) no testemunho SAT-073 demonstra que os cocolitoforídeos podem ser responsáveis por parte do aporte de carbonato para o fundo marinho na região estudada. Entretanto, as curvas destes dois parâmetros não se apresentam de forma coerente para todo o período.

Entre 37,91 ka e 18,98 ka, o aporte de carbonato é relativamente baixo, e a curva de abundância absoluta apresenta uma oscilação pouco relevante, mas, em

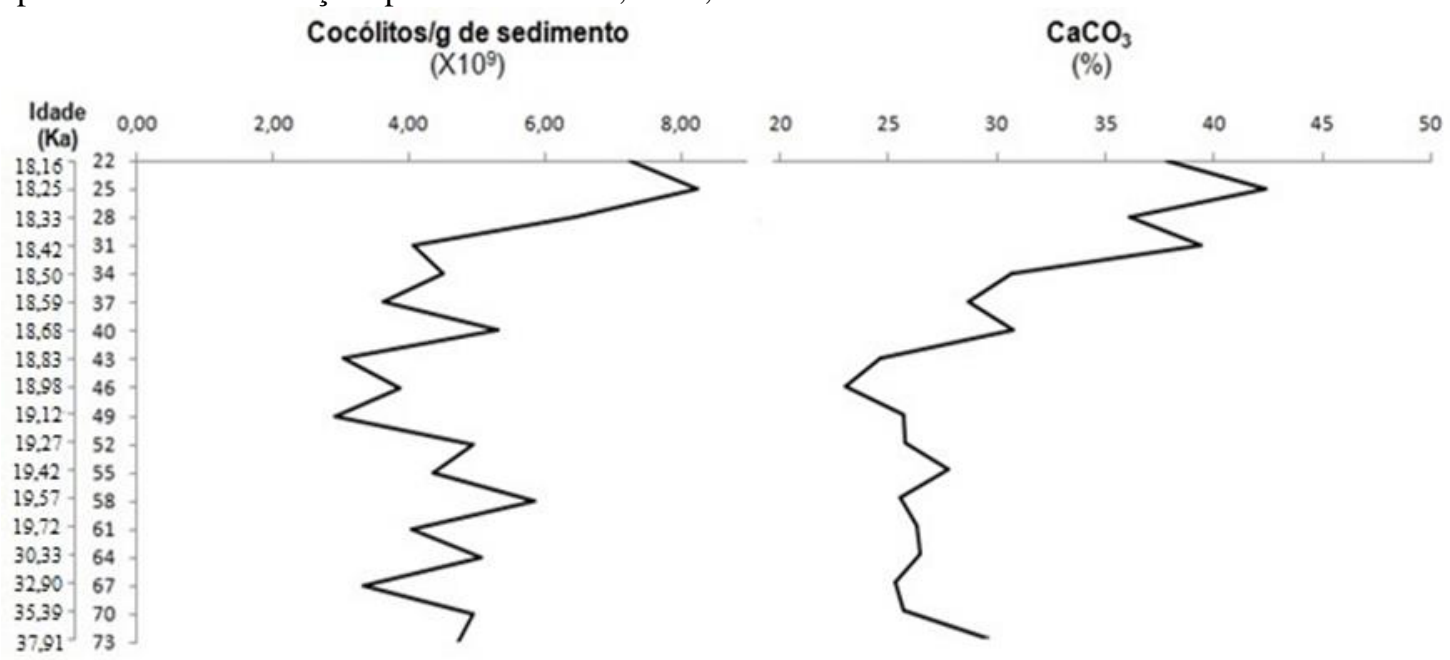
(X10 $10^{9}$

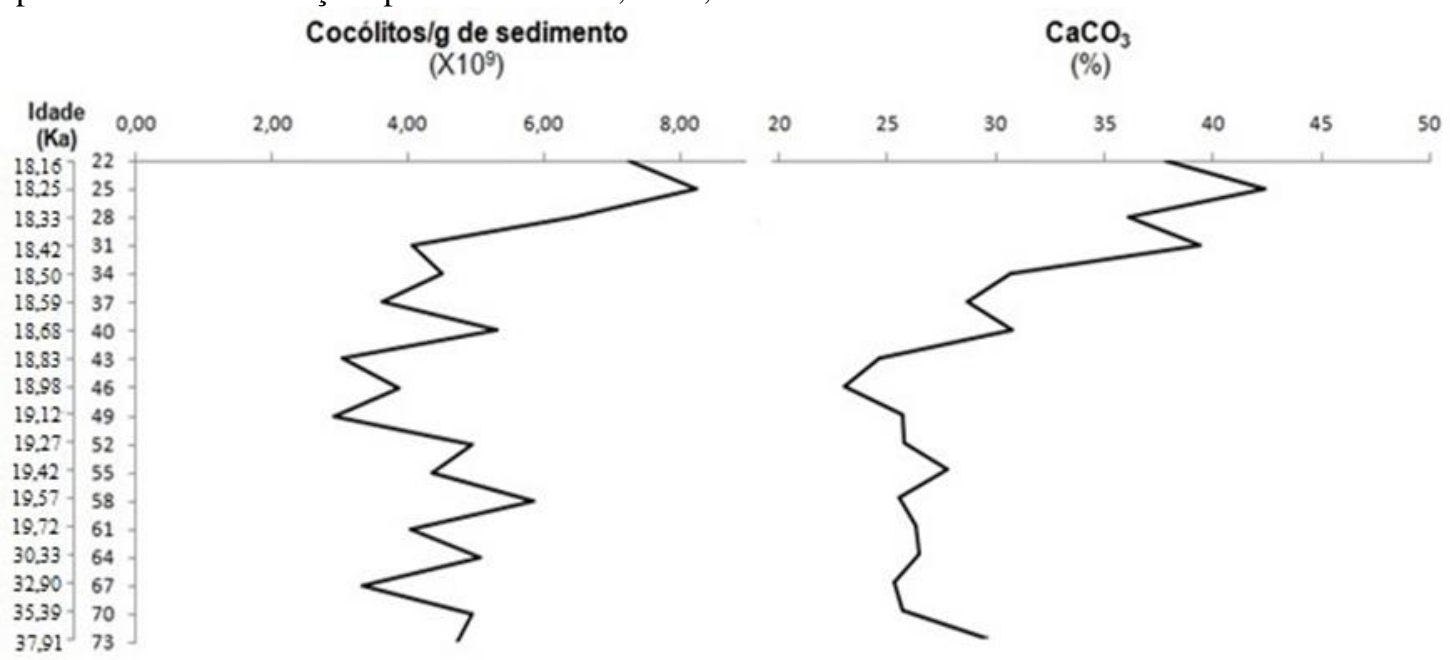

geral, também é relativamente baixa. A partir de 19,27 ka nota-se uma diminuição na curva de abundância absoluta, ainda condizente com os valores de carbonato. Portanto, há um menor aporte de carbonato para o fundo marinho no intervalo mais próximo ao UMG (entre 19,72 e 19,12 ka), com grande participação dos cocolitoforídeos neste aporte. Neste intervalo também foi constatada uma maior representatividade dos cocolitoforídeos na produtividade da região.

Figura 2: Relação da abundância absoluta de cocólitos com o conteúdo de $\mathrm{CaCO}_{3}$ através do tempo no testemunho SAT-073.

Eventos de regressão marinha podem liberar carbono orgânico preso em reservatórios efêmeros causando um aumento da bomba biológica, e resultando em uma diminuição do pH oceânico, levando assim, a uma maior dissolução de $\mathrm{CaCO}_{3}$ durante os períodos glaciais (Crowley 1983, Baumannet al. 2004). Desta maneira, é esperado que maiores teores de carbonato nos sedimentos marinhos ocorram em períodos de maior aquecimento, como no início da deglaciação. No testemunho SAT-073, observa-se uma elevação no aporte de $\mathrm{CaCO}_{3}$ para o sedimento há $18,83 \mathrm{ka}$, paralelamente à curva de abundância absoluta de cocólitos.

Boeckel \& Baumann (2004), investigando a distribuição de cocólitos nos sedimentos superficiais do Atlântico Sudeste, relacionaram uma maior contribuição dos cocolitoforídeos para o aporte de $\mathrm{CaCO}_{3}$ com áreas de maior produtividade, associadas à Corrente da Benguela. Em áreas de oceano profundo, cocolitoforídeos não tiveram um papel preponderante, devido ao baixo valor de carbonato encontrado. Os menores valores para o $\mathrm{CaCO}_{3}$, foram encontrados em áreas próximas ao continente. Apesar de algumas amostras terem sido afetadas pela dissolução, a distribuição dos cocolitoforídeos nos sedimentos superficiais refletiram as diferentes condições oceanográficas da superfície.

Em águas profundas, os cocolitoforídeos são mais resistentes à dissolução que os foraminíferos Hay (2004), grupo que também possui grande importância no aporte de carbonatos. Isto ocorre devido ao transporte acelerado dos cocólitos para o fundo, via afundamento de pelotas fecais, Honjo (1976). Esses fatores, juntamente com a pouca dissolução constatada na área de estudo, indicam os cocolitoforídeos como os principais contribuintes no aporte de $\mathrm{CaCO}_{3}$ nos sedimentos do fundo oceânico da região, durante o UMG e início da deglacioção.

Espécies como Gephyrocapsa spp., Emiliania huxleyi e Florisphaera profunda, são as maiores responsáveis pelo aporte de $\mathrm{CaCO}_{3}$ mesmo possuindo cocólitos pequenos, já que suas porcentagens foram muito maiores que as porcentagens das demais espécies encontradas no registro. As curvas de abundância absoluta de Florisphaera profunda e Emiliania huxleyi, aumentam a partir de 18,5 ka e 18,33 ka respectivamente, ao passo que Gephyrocapsa spp. tem sua abundância absoluta diminuída a partir de 18,33 ka (figura 3). Vale salientar que esta diminuição na abundância de Gephyrocapsa spp. está de acordo com o aumento em E. huxleyi, porque estes táxons sucedem-se em termos da ocupação de nichos, já que E. huxleyi é considerada como o membro recente da linhagem evolutiva de Gephyrocapsa spp., Baumann \& Freitag (2004).

Os táxons Emiliania huxleyi e Gephyrocapsa spp., se somados, são na maioria dos casos, absolutamente mais abundantes que $F$. profunda que, além disso, possui cocólitos muito pequenos. Os cocólitos de Gephyrocapsa apresentam grande contribuição para o 
aporte de carbonato para o fundo oceânico até a metade do testemunho, há $18,98 \mathrm{ka}$, a partir de onde E. huxleyi passa a ter a maior expressividade. Os cocolitoforídeos são considerados como os organismos calcários mais produtivos do mundo, possuindo um importante papel no ciclo de carbono global. A formação de esqueletos de calcita nas camadas superficiais do oceano e o seu afundamento subsequente para maiores profundidades modifica a alcalinidade oceânica superficial, afetando diretamente a trocas de $\mathrm{CO}_{2}$ entre oceano e atmosfera, Rost \& Riebesell (2004). Atualmente, E. huxleyi, que é extremamente cosmopolita, é a principal espécie formadora de blooms nos oceanos, Tyrrell \& Merico (2004), sendo a principal responsável pela participação dos cocolitoforídeos no ciclo do carbono. As espécies de Gephyrocapsa eram globalmente dominantes durante intervalos do Pleistoceno, e assim, devem ter sido um importante componente no ciclo de carbono global, com importância análoga à atual de E. huxleyi, Bollmann (1997).

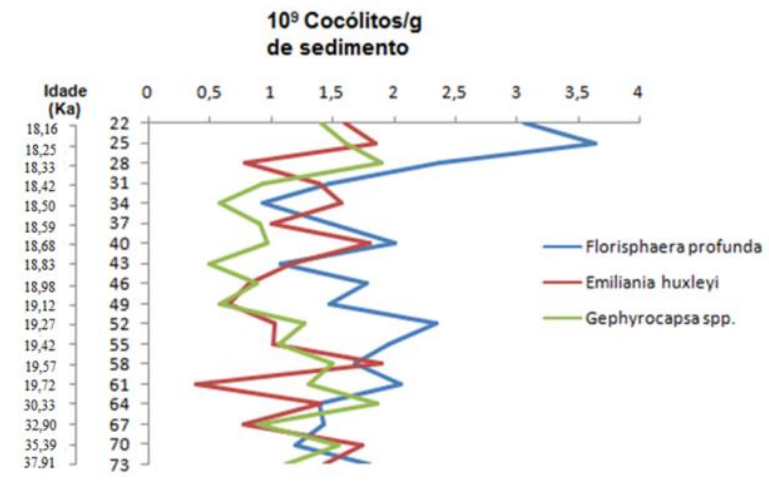

Figura 3: Relação entre as abundâncias absolutas dos cocolitoforídeos contribuintes para o aporte de $\mathrm{CaCO}_{3}$ para o fundo marinho através do tempo no testemunho SAT-073.

\section{Conclusões}

Durante o UMG a transferência de $\mathrm{CaCO}_{3}$ para o fundo marinho foi considerada baixa (em comparação com a da deglaciação), embora tenha-se constatado uma boa contribuição dos cocolitoforídeos para o aporte de carbonato durante este período;

As espécies oportunistas Emiliania huxleyi e Gephyrocapsa spp. são as maiores contribuintes entre os cocolitoforídeos para o aporte de $\mathrm{CaCO}_{3}$ na região.

\section{Referências}

Antunes R.L. 2007. Nanofósseis Calcários do Quaternário Tardio da Margem Continental Brasileira. Ciência técnica petróleo, Rio de Janeiro, 76p.

Bastos C.C., Ferreira N.J., 2000. Análise Climatológica da Alta Subtropical do Atlântico Sul. Anais do XI Congresso Brasieleiro de Meteorologia, Rio de Janeiro. Sociedade Brasileira de Metereologia, 612-619p.

Baumann K.H., Böeckel B., Frenz M. 2004. Coccolithcontribution to South Atlantic carbonate sedimentation. In: Thierstein H.R., Young J.R., (eds.)CoccolithophoresFrom Molecular Processes to Global Impact. Springer-Verlag, Berlin Heidelberg, part. 3: 367$401 p$.

Baumann K.H., Freitag T. 2004. Pleistocene fluctuations in the northern Benguela Current system as revealed coccolith assemblages. Marine Micropaleontology, 52: 192-215.
Boeckel B., Baumann K.H. 2004. Distribution of coccoliths in surface sediments of the south-eastern South Atlantic Ocean: ecology, preservation and carbonate contribution. Marine Micropaleontology, 51: 301-320.

Bollmann J. 1997. Morphology and biogeography of Gephyrocapsacoccoliths in Holocene sediments. Marine Micropaleontology, 29: 319-350.

Crowley T.J. 1983. Calcium-carbonate preservation patterns in the Central North Atlantic during the last 150,000 years. Marine Geology, 51: 1-14.

Degens E.T., Kempe S., Richey J.E. 1991. Scoope 42: Biogeochemistry of major world rivers. Wiley, UK.

Dias J.L., Sad A.R.E., Fontana R.L., Feijó F.J. 1994. Bacia de Pelotas. Boletim de Geociências da Petrobrás. Rio de Janeiro, 8: 235-245.

Hay W.W. 2004. Carbonatefluxesandcalcareousnannoplankton. In: Coccolithophores From Molecular Processes to Global Impact. Springer-Verlag, Berlin Heidelberg, part. 4: 509-528.

Heinze C. 1994. Glacial ocean carbon cycle modeling. In: Zahn R., Pedersen T.F., Kaminski M.A., Labeyrie L. (eds) Carbon Cycling in the Glacial Ocean: Constrains on the Ocean's Role in Global Change. Springer-Verlag, Berlin Heidelberg, 15-39p.

Honjo S. 1976. Coccoliths: Production, transportation and sedimentation. Marine Micropaleontology, 1: 65-79.

Koch C., Young J.R. 2007.A simple weighing and dilution technique for determining absolute abundance of coccoliths from sediment samples. Journal of Nannoplankton Research, 29:67-69.

Mix A.C., Bard E., Schneider R. 2001. Environmental processes of the ice age: land, oceans, glaciers (EPILOG). Quaternary Science Reviews, 20: 627-657.

Möller O.O. Jr., Piola A.R., Freitas A.C., Campos E.J.D. 2008. The effects of river discharge and seasonal winds on the shelf off southeastern South America. Continental Shelf Research, 28: 1607-1624.

O’Dea S., Gibbs S.J., Bown P.R., Young J.R., Poulton A.J., Newsam C., Wilson P.A. 2014. Coccolithophores calcification response to past ocean acidification and climate change. Nature Communications, 5: 1-7.

Piola A.R., Matano R.P., Palma E.D., Möller O.O. Jr. 2005. The influence of the Plata River discharge on the western South Atlantic shelf.GeophysicalResearchLetters, 32: 1-4.

Rost B., Riebesell U. 2004. Coccolithophore calcification and the biological pump: response to environmental changes. In: Thierstein H.R., Young J.R., (eds.) Coccolithophores From Molecular Processes to Global Impact. Springer-Verlag, Berlin Heidelberg, part.2: 99-124p.

Stuiver M., Reimer P.J. 1993. Extended 14C data base and revised Calib 3.0 14C age calibration program. Radiocarbon, 35: 215230.

Tyrrell T., Merico A. 2004. Emilianiahuxleyi: Bloom observations and the conditions that induce them. In: Thierstein H.R., Young J.R., (eds.),CoccolithophoresFrom Molecular Processes to Global Impact. Springer-Verlag, Berlin Heidelberg, part. 3: 367401p.

Wainer I., Taschetto A.S. 2006. Climatologia na região entre o Cabo de São Tomé (RJ) e o Chuí (RS). Diagnóstico para os períodos relativos aos levantamentos pesqueiros do Programa REVIZEE. In: Rossi-Wongtschowski C.L.B., Madureira L.S.P. (Eds). O ambiente oceanográfico da plataforma continental e do talude na região sudeste-sul do Brasil. Editora Universidade de São Paulo, São Paulo 1: 121-160p. ${ }^{\text {i }}$

Recebido 15 de junho de 2016 Aceito 01 de agosto de 2016 